\title{
Metodologia e produzione delle Linee Guida
}

\author{
Francesco Burrai ${ }^{1}$, Margherita Gambella ${ }^{2}$, Angelica Scarpa ${ }^{2}$, Stefano Cabula ${ }^{3}$ \\ ${ }^{1}$ SC Formazione, Ricerca e Cambiamento Organizzativo, ATS Sardegna, Sassari - Italy \\ ${ }^{2}$ Università degli studi di Sassari, Sassari - Italy \\ ${ }^{3}$ Assessorato dell'igiene e della sanità e dell'assistenza sociale. Regione Autonoma della Sardegna, Cagliari - Italy
}

\begin{abstract}
Methodology and production of guidelines
This article has the aim of informing the reader about methodology and production of Clinical Practice Guidelines (CPG) and the instruments in order to be able to approach the whole process, from the production to the publication and distribution, but also to updated CPG. The importance of the issue is given by: 1 ) the need to avoid the CPG duplication, which is the reason of waste of resources and that raises confusion among users; 2 ) the need to ensure the independence of the experts involved and without conflict of interests; 3 ) the use of methodologies that respect the best quality standards; 4) the inhomogeneity of healthcare processes in the Italian territory and practices not based on scientific evidences; 5 ) the consultation and the commentary of stakeholders on the recommendations produced.
\end{abstract}

Keywords: Clinical Practice Guidelines, Dissemination, Evidence, Guideline, Methodology

\section{Introduzione}

L'obiettivo di questo articolo è quello di avvicinare gli operatori sanitari all'utilizzo delle Linee Guida (LG) nella pratica clinica non solo per migliorare la qualità dell'assistenza, ma soprattutto per aiutare i professionisti della salute a prendere decisioni cliniche basate sulle evidenze scientifiche. Le LG sono "documenti che contengono raccomandazioni finalizzate a migliorare l'assistenza ai pazienti, basate su una revisione sistematica delle evidenze e sulla valutazione di benefici e rischi di opzioni alternative" (1). Sono nate alla fine degli anni '80, quando, dopo studi e ricerche scientifiche, si è sentito il bisogno di riportare le nuove conoscenze nella pratica clinica (2). Conoscere le LG, sapendo come si sviluppano e come si aggiornano nel tempo, permette agli operatori di rimanere costantemente aggiornati sull'evoluzione delle cure sanitarie, aspetto fondamentale, visti i continui sviluppi in questo ambito.
Received: April 20, 2021

Accepted: October 6, 2021

Published online: October 29, 2021

Indirizzo per la corrispondenza:

Francesco Burrai

SC Formazione, Ricerca e Cambiamento Organizzativo

ATS Sardegna, Sassari - Italy

francesco.burrai@atssardegna.it

\section{Linee Guida in sanità}

Il processo di produzione delle LG ha origine da una revisione sistematica della letteratura. Questa rappresenta un metodo di sintesi delle migliori evidenze scientifiche disponibili (3), ottenute tramite la consultazione di banche dati attraverso le quali vengono selezionati trial clinici che rappresentano i gold standard per valutare l'efficacia degli interventi riguardanti un determinato quesito clinico (4). Le raccomandazioni sviluppate vengono, poi, adattate e applicate al contesto locale attraverso i Percorsi Diagnostico-Terapeutici-Assistenziali (PDTA). In questa fase, tutto ciò che sembrava solo teoria diviene pratica e le Linee Guida vengono adattate al singolo paziente. I PDTA, in base alla loro complessità, sono costituiti da uno o più processi assistenziali. La sequenza di azioni effettuate dai professionisti per erogare l'assistenza all'interno dei processi assistenziali prende il nome di procedura. Quando si parla di protocollo, si intende un qualcosa di prescrittivo e vincolante dal punto di vista giuridico, che obbliga i sanitari ad applicarlo a tutti i pazienti. Il protocollo può riguardare l'intero PDTA, un processo assistenziale o una procedura (4).

\section{Linee Guida e responsabilità}

Le LG in Italia assumono particolare rilevanza dal punto di vista giuridico a seguito della pubblicazione della legge n. 24 dell'8 marzo 2017 (5). Questa legge prevede che gli esercenti le professioni sanitarie si attengano alle LG elaborate da enti pubblici e privati autorizzati dal Ministero della Salute secondo D.M. 2.08.2017 (6) e che, in mancanza di LG, 
si debbano attenere alle buone pratiche clinico-assistenziali. Inoltre, come citato dall'Art. 6 della stessa legge, l'esercente che si attiene alle raccomandazioni, in caso di imperizia, non è punibile (5). A seguito di questa legge, nasce il Sistema Nazionale Linee Guida (SNLG) dell'Istituto Superiore di Sanità (ISS), all'interno del quale vengono pubblicati le $L G$ e i relativi aggiornamenti, disponibili per tutti i sanitari (7).

\section{Il processo di produzione delle Linee Guida}

Per lo sviluppo delle LG sono presenti sei stadi. II primo stadio è caratterizzato dalla ricerca di informazioni per la definizione dello "scope", come informazioni sulla pratica clinica corrente e sui pareri e sulle esperienze di esperti, pazienti e cittadini. Nel secondo stadio, si svolge l'analisi delle ragioni per lo sviluppo delle raccomandazioni. Nel terzo stadio, si procede con l'identificazione della popolazione e degli aspetti più incisivi dell'argomento che permette di migliorare le decisioni nelle fasi di cura da parte dei professionisti della salute. Durante il quarto stadio, avviene la verifica da parte degli stakeholder, quali società scientifiche, di pazienti o caregiver, sulla scelta della popolazione delle LG e degli elementi chiave. La quinta è la fase della consultazione dello "scope" fatta dagli stakeholder tramite dei moduli standardizzati. Infine, nel sesto stadio, avviene la finalizzazione dello "scope", dove vengono valutati altri aspetti non considerati in precedenza, come altri gruppi di popolazione o aspetti assistenziali. Al termine delle fasi, lo "scope" viene pubblicato e non potrà più essere modificato. Per poter dare inizio al processo di produzione delle LG, viene prima costituito un gruppo di lavoro con competenze multidisciplinari e multiprofessionali. Nel team sono presenti diverse figure: 1) Developer, che sono uno o più esperti che redigono lo "scope" e l'ambito di interesse scelto e che formulano i quesiti; 2) Evidence Review Team, esperti di revisione sistematica della letteratura; 3 ) Team per l'analisi economica; 4) Documentalisti, Esperti di bioetica e Stakeholder, chiamati per esprimersi sullo "scope" e sull'ambito di interesse della LG (8). La presenza di varie figure all'interno del team multidisciplinare garantisce un'analisi dello "scope" da diversi punti di osservazione e fornisce un contributo importante a quello che sarà il contenuto della LG.

\section{Disclosure e gestione dei conflitti di interesse}

Nel processo di sviluppo delle LG, i membri coinvolti devono garantire che i propri interessi privati e personali non interferiscano con il ruolo che ricoprono nello svolgimento del processo. Vengono definite conflitti di interesse, Conflict Of Interest (COI), tutte quelle "circostanze che espongono al rischio che il giudizio professionale o le azioni riguardanti un interesse primario vengano indebitamente influenzati da un interesse secondario" (9). I COI possono essere diretti o indiretti: i primi rappresentano interessi prettamente economici, i secondi, invece, rappresentano gli avanzamenti di carriera o il prestigio sociale (10). Per questo motivo, tutti i membri coinvolti nella produzione della LG sono tenuti a garantire la loro imparzialità dichiarando pubblicamente i propri $\mathrm{COI}$, in modo che ci sia più trasparenza possibile e che questi possano essere gestiti al fine di evitare bias. Di fatto, la dichiarazione di un COI non implica obbligatoriamente la cessazione dell'incarico, ma, sulla base di un'attenta valutazione che avviene caso per caso, ci si può trovare di fronte a tre situazioni principali: è sufficiente una dichiarazione pubblica dei COI senza limitazioni alla partecipazione al lavoro, il membro può essere limitato parzialmente oppure può essere totalmente escluso. Le decisioni prese in merito alla dichiarazione e alla gestione dei COI devono essere riportate nella documentazione finale della LG (11).

\section{Metodologia di produzione delle Linee Guida: il GIN}

II GIN è un network nato nel 2002 che ha proposto dei requisiti condivisi a livello internazionale, fondamentali nella metodologia di produzione per LG di qualità. Un vantaggio importante riguardante questi requisiti sta nel fatto che possono essere rispettati anche da organizzazioni produttrici di LG che dispongono di un budget non elevato. II metodo utilizzato per il soddisfacimento di ogni requisito deve essere riportato nella documentazione della LG. I requisiti identificati sono 11 e possono, eventualmente, essere riadattati a livello locale. Secondo il GIN, il gruppo di sviluppo della LG dovrebbe essere composto da circa 10-20 componenti, tra i quali, oltre ai soggetti esperti dell'argomento, anche i pazienti. II processo di raggiungimento del consenso da parte dei membri del team dovrebbe essere definito prima dell'inizio dello sviluppo della LG e documentato. Ogni LG dovrebbe riportare la disclosure dei COI. Una LG di qualità dovrebbe definire in modo chiaro gli obiettivi e gli ambiti di applicazione della stessa, descrivere in maniera precisa il processo di produzione e utilizzare metodi sistematici per revisionare e identificare le migliori evidenze scientifiche sulle quali verrà basata la raccomandazione che deve essere espressa, considerando anche benefici, rischi e costi. Per il grading delle evidenze e per esprimere la forza delle raccomandazioni dovrebbero essere utilizzati sistemi standardizzati e uno strumento utile a questo scopo è il sistema Grading of Recommendations Assessment, Development, and Evaluation (GRADE). Una volta sviluppata la LG, prima di essere pubblicata, dovrebbe essere revisionata da stakeholder esterni. Le evidenze scientifiche, nel corso del tempo, possono variare, per questo motivo in ogni LG si dovrebbe specificare il tempo di validità e il metodo da utilizzare per l'aggiornamento delle raccomandazioni. Infine, una LG dovrebbe dichiarare finanziamenti e sponsor (12).

\section{Formulazione dei quesiti}

I quesiti delle LG sono formulati sulla base dell'acronimo PICO. Questo definisce la popolazione di interesse, 
l'intervento che si vuole effettuare, l'intervento alternativo o il non intervento e gli outcome rilevanti per ciascun quesito, che verranno valutati per la formulazione della raccomandazione. Secondo il metodo GRADE, gli outcome vengono identificati e classificati in base alla loro importanza tramite una votazione da parte di ciascun membro del team. La media o mediana del voto ottenuto di quello specifico outcome permetteranno di identificarlo come critico, importante ma non essenziale o non importante. Solo i critici verranno considerati nella formulazione delle raccomandazioni (8).

\section{Revisione sistematica della letteratura, valutazione critica e selezione degli studi}

Le raccomandazioni sono basate su una sintesi delle migliori evidenze disponibili attuali. II processo di ricerca delle evidenze viene fatto tramite la consultazione di banche dati attraverso le quali vengono selezionati studi di qualità pertinenti ai quesiti. Le evidenze derivanti dagli studi vengono, poi, riassunte in evidence profile e in tabelle che riportano la qualità e il riepilogo dei risultati per ciascun outcome considerato essenziale o critico. In base alla loro qualità, le prove possono essere classificate in 4 categorie: alta, moderata, bassa, molto bassa. Secondo il metodo GRADE la qualità viene valutata considerando non solo il disegno dello studio, ma anche altri fattori che possono aumentare o diminuire il grado di qualità. Studi sperimentali come gli studi randomizzati controllati forniscono una qualità delle evidenze alta, mentre studi osservazionali, come i case report, forniscono una qualità delle evidenze bassa. I fattori che possono modificare il grado di qualità sono, per esempio, la corrispondenza dei risultati nei diversi studi, la precisione dei risultati e la qualità metodologica di conduzione dello studio. Una volta terminata la classificazione, viene eseguita la valutazione globale della qualità delle prove e la valutazione costo-efficacia dell'intervento preso in considerazione (8).

\section{GRADE-ADOLOPMENT}

La produzione di raccomandazioni può avvenire attraverso tre modalità, che possono essere: adottate, adattate o sviluppate ex novo. II metodo GRADE-ADOLOPMENT è un metodo che consente agli sviluppatori di LG di stabilire con quale delle tre modalità svilupparle. II metodo più veloce fra i tre è l'adozione. In questo caso, la LG viene analizzata dai membri del team che decidono di utilizzarla così come è stata prodotta dagli sviluppatori originali, senza apporre alcuna modifica e fornendo informazioni sull'implementazione. Una LG adattata invece presenta dei cambiamenti che possono riguardare una o più raccomandazioni rispetto alla LG originale. Per lo sviluppo di una LG ex novo, si intende la formulazione di nuove domande e, solo nel caso in cui non si riscontrino in letteratura LG con raccomandazioni corrispondenti, la LG verrà sviluppata ex novo (13).

\section{Formulazione delle raccomandazioni e metodo GRADE}

Per la formulazione di una raccomandazione viene utilizzato il metodo GRADE. Dopo aver valutato la qualità delle prove, sulla base delle stesse viene stabilita la forza (grado di fiducia) della raccomandazione, espressa attraverso termini standard: forte, debole, condizionata a favore o condizionata contro (14). Nelle raccomandazioni forti, si utilizza il termine standard "si raccomanda" o "non si raccomanda", a seconda che la raccomandazione sia a favore dell'intervento o contro di esso. Le raccomandazioni condizionate sono quelle per cui esiste ancora incertezza sugli effetti e sono formulate con il termine standard "si suggerisce" o "non si suggerisce". Per ogni raccomandazione, si devono includere anche la giustificazione e le informazioni sull'implementazione (8).

\section{Revisione esterna della Linea Guida}

L'ultima fase prima della pubblicazione della LG consiste nella valutazione della stessa da parte di tre esperti esterni. Questi valutano i contenuti della $L G$ e la qualità del reporting e della metodologia. A seguito della valutazione, in caso di esito insoddisfacente, i produttori di LG hanno 60 giorni di tempo per effettuare le dovute modifiche e, superato questo limite, la LG non può più essere ammessa (8).

\section{Editing, pubblicazione e disseminazione della Linea Guida}

La versione finale della LG viene pubblicata sul sito SNLG e, in seguito, "pubblicizzata" dall'ufficio stampa dell'ISS tramite comunicato stampa, newsletter e social media. Possono essere anche organizzate delle conferenze stampa utili per analizzare e discutere con gli sviluppatori stessi i diversi aspetti della LG. Di fondamentale importanza è informare anche coloro che ricevono in prima persona gli interventi descritti nelle raccomandazioni; vengono, infatti, prodotte e pubblicate delle versioni di LG adatte alla comprensione di pazienti e cittadini $(8,15)$.

A seguito della variabilità delle metodologie di produzione delle $L G$, che si riflettono in una variabilità della loro qualità, sono stati generati alcuni strumenti standard internazionali che verranno descritti qui di seguito.

\section{Appraisal of Guidelines for Research \& Evaluation II}

Appraisal of Guidelines for Research \& Evaluation II (AGREE II) è uno strumento che rappresenta un punto di riferimento a livello internazionale utile per valutare la qualità delle LG. Creato dall'AGREE Collaboration nel 2003 e successivamente migliorato e riadattato in alcune delle sue parti, consiste in una checklist che comprende 23 item raccolti in 6 dimensioni. Queste ultime riguardano gli obiettivi e i campi di applicazione della $L G$, il coinvolgimento degli stakeholder, 
il rigore metodologico, la chiarezza espositiva, l'applicabilità e l'indipendenza editoriale. In aggiunta a questi, sono previsti anche 2 item di valutazione complessiva, utili a fornire un punteggio sulla qualità della $L G$ e a raccomandarne o meno I'utilizzo. Ad ogni item viene assegnato un punteggio da 1 a 7. È prevista l'assegnazione del punteggio 1 per tutti quegli item di cui mancano informazioni, mentre il punteggio 7 è fissato per quegli item che presentano informazioni dettagliate. Viene, poi, calcolato uno score di qualità indipendente per ciascuna dimensione, attraverso la somma degli score degli item che compongono quella specifica dimensione. A oggi, non essendo stati identificati degli score minimi di qualità per le dimensioni, non è possibile basarsi su questi per definire se una LG è di alta qualità o meno; piuttosto, questi score risultano utili per confrontare diverse LG tra loro. AGREE II è uno strumento molto utile sia per gli utilizzatori delle LG, per capire o meno se adottare le raccomandazioni presenti in esse, sia per chi le produce, in quanto rappresenta una guida per sviluppare con rigore metodologico le LG. Un limite all'utilizzo di questo strumento sta nel fatto che non è adatto per la valutazione delle guidance (16).

\section{AGREE Global Rating Scale}

Un altro strumento è l'AGREE Global Rating Scale (GRS), più semplice e veloce da utilizzare rispetto all'AGREE II. È costituito da 4 item che riguardano il processo di sviluppo, la modalità di presentazione dei contenuti, la completezza del reporting e la validità clinica. Come per l'AGREE II, a ognuno di questi item viene assegnato un punteggio da 1 a 7 , dove 1 rappresenta la qualità minima e 7 la qualità massima. Una volta assegnato il punteggio per ognuno degli item, si procede alla compilazione della valutazione complessiva della LG, che considera la qualità e l'indicazione o meno all'utilizzo della stessa nella pratica clinica come punto di riferimento sul quale basare le proprie decisioni professionali (17).

\section{AGREE Reporting Checklist}

Uno strumento standard utilizzato a livello internazionale per migliorare la qualità del reporting delle LG è l'AGREE Reporting Checklist (18). Analogamente ad AGREE II, è costituito da 23 item raccolti in 6 dimensioni, con la differenza che, nell'AGREE Reporting Checklist, gli item sono più improntati alla verifica della completezza delle informazioni riportate, piuttosto che alla qualità delle stesse. Questo strumento si rivela utile durante il processo di stesura, ma, anche a LG completata, per verificare che tutte le informazioni siano correttamente riportate (19).

\section{Aggiornamento delle Linee Guida e checklist check-up}

Per far sì che le raccomandazioni siano sempre basate sulle migliori evidenze, ogni LG possiede un periodo massimo di validità di 3 anni. Al termine di questo lasso di tempo, dovrebbe essere effettuata nuovamente una revisione sistematica della letteratura per verificare la validità o meno delle evidenze a supporto delle raccomandazioni. Nel caso in cui, a seguito di questa revisione, siano disponibili nuove evidenze che possano influenzare la raccomandazione, è necessario ricorrere a un aggiornamento della LG, che può essere completo o parziale. Una LG totalmente aggiornata ha nuove raccomandazioni basate su nuove evidenze e viene pubblicata nel sito web del SNLG in sostituzione della LG precedente. Le LG parzialmente aggiornate sono incluse in un'unica pubblicazione insieme alla versione precedente, sottolineando le parti aggiornate e permettendo agli utilizzatori di riconoscerle. Per le LG aggiornate, è stato sviluppato un checklist check-up, uno strumento costituito da 16 item, utile sia per coloro che si devono occupare dell'aggiornamento sia per gli utilizzatori della LG aggiornata. II check-up non valuta la qualità dei processi di aggiornamento, piuttosto considera la completezza del reporting, il rigore metodologico e la presentazione della LG aggiornata (20).

\section{Conclusioni}

In un periodo in cui la scienza fa passi da gigante, le LG sono un punto di connessione e di supporto per i professionisti sanitari di tutto il mondo. Questi, in ragione della loro autonomia professionale e della loro responsabilità, utilizzano le LG come sostegno alle scelte clinico-assistenziali, adattando le raccomandazioni al singolo e fornendo cure standardizzate e un'assistenza di qualità centrata sulla persona. Al giorno d'oggi, i pazienti risultano essere molto più interessati riguardo alle pratiche sanitarie rispetto al passato e anch'essi possono prendere visione delle raccomandazioni presenti nelle LG, con la speranza che i professionisti di oggi siano sempre più interessati e propensi all'applicazione e alla stesura delle LG e facendo parte delle associazioni che le producono, per andare a risolvere tutti gli aspetti dell'assistenza che non presentano evidenze. L'invito a interessarsi alle LG è rivolto anche agli studenti universitari in ambito scientificosanitario per approcciarsi fin da subito a ciò con cui avranno a che fare nel loro futuro professionale.

\section{Disclosures}

Conflict of interest: The authors declare no conflict of interest. Financial support: This research received no specific grant from any funding agency in the public, commercial, or not-for-profit sectors.

\section{Bibliografia}

1. Cartabellotta A. Standard internazionali per la produzione delle linee guida. Giornale Italiano di Ortopedia e Traumatologia. 2016;42:376-383. Online (ultimo accesso Aprile 2021)

2. Il programma nazionale delle linee guida tra passato e presente. Istituto superiore di sanità. Online (ultimo accesso Luglio 2021) 
3. Sala V, Moja L, Moschetti I, Bidoli S, Pistotti V, Liberati A. Revisioni sistematiche - Breve guida all'uso. Centro Cochrane Italiano; 2006.

4. Cartabellotta A. Linee guida, percorsi, processi, procedure, protocolli. II caos regna sovrano: è tempo di mettere ordine! GIMBE news 2008;1:4-5. Pubblicato: 9 dicembre 2008. Online (ultimo accesso Ottobre 2021)

5. Legge 8 marzo 2017, n. 24, "Disposizioni in materia di sicurezza delle cure e della persona assistita, nonché in materia di responsabilità professionale degli esercenti le professioni sanitarie". Online (ultimo accesso Ottobre 2021)

6. Decreto 02 agosto 2017, "Elenco delle società scientifiche e delle associazioni tecnico-scientifiche delle professioni sanitarie". Online (ultimo accesso Ottobre 2021)

7. Sistema Nazionale Linee Guida dell'Istituto Superiore di Sanità. Online (accesso Luglio 2021)

8. Manuale operativo. Procedure per la proposta di inserimento di Linee Guida del Sistema Nazionale Linee Guida. Centro Nazionale Eccellenza Clinica, Qualità e Sicurezza delle Cure. Versione 2.0 20-04-2018. Online (ultimo accesso Ottobre 2021)

9. Institute of Medicine. Conflict of interest in medical research, education, and practice. Washington, DC: National Academies Press; 2009.

10. Schünemann HJ, Osborne M, Moss J, et al; ATS Ethics and Conflict of Interest Committee and the Documents Development and Implementation Committee. An official American Thoracic Society Policy statement: managing conflict of interest in professional societies. Am J Respir Crit Care Med. 2009;180(6): 564-580. CrossRef PubMed

11. Schünemann HJ, Al-Ansary LA, Forland F, et al. Disclosure e gestione dei conflitti di interesse nelle linee guida: i principi del Guidelines International Network. Evidence 2016;8(3): e1000136. CrossRef
12. Qaseem A, Forland F, Macbeth F, et al. Guidelines International Network: verso standard internazionali per la produzione di linee guida. Evidence. 2012;4(6):e1000022. CrossRef

13. Schünemann HJ, Wiercioch W, Brozek J, et al. GRADE Evidence to Decision (EtD) frameworks for adoption, adaptation, and de novo development of trustworthy recommendations: GRADEADOLOPMENT. J Clin Epidemiol. 2017;81:101-110. CrossRef PubMed

14. Guyatt G, Oxman AD, Akl EA, et al. GRADE guidelines: 1. Introduction-GRADE evidence profiles and summary of findings tables. Journal of Clinical Epidemiology 64(2011):383e394. CrossRef PubMed

15. Alonso-Coello $P$, Schünemann $\mathrm{HJ}$, Moberg J, et al. GRADE Evidence to Decision (EtD) frameworks: a systematic and transparent approach to making well informed healthcare choices. 1: Introduction. BMJ 2016;353:i2016. CrossRef

16. AGREE Next Step Consortium. AGREE II. Checklist per valutare la qualità delle linee guida. Fondazione GIMBE: Bologna, aprile 2011. Online (Ultimo accesso Aprile 2021)

17. Brouwers $M C$, Kho ME, Browman GP, et al. The global rating scale complements the AGREE II in advancing the quality of practice guidelines. J Clin Epidemiol 2012;65(5):526-34. CrossRef PubMed

18. Brouwers MC, Kerkvliet K, Spithoff K; AGREE Next Steps Consortium. The AGREE Reporting Checklist: a tool to improve reporting of clinical practice guidelines. BMJ. 2016;352:i1152. CrossRef PubMed

19. Cartabellotta A, Laganà AS. AGREE Reporting Checklist: uno strumento per migliorare il reporting delle linee guida Evidence 2016;8(7):e1000146. CrossRef

20. Vernooij RWM, Alonso-Coello P, Brouwers M, et al. Reporting Items for Updated Clinical Guidelines: Checklist for the Reporting of Updated Guidelines (CheckUp). PLOS Medicine 2017;14(1):e1002207. CrossRef 